\title{
NASAL MUCOSA AND MUCOCILIARY CHANGES IN EXTREME HOT AND DRY WEATHER IN THE DESERTS
}

\author{
Sanajeet Kumar Singh ${ }^{1}$, Suresh Mokamati², Manoj Kumar Kanzhuly33, Nikhil Kumar ${ }^{4}$ \\ ${ }_{1}^{1}$ Professor, Department of ENT, Head and Neck Surgery, AFMC, Pune. \\ ${ }^{2}$ Resident, Department of ENT, Head and Neck Surgery, AFMC, Pune. \\ ${ }^{3}$ Assistant Professor, Department of ENT, Head and Neck Surgery, AFMC, Pune. \\ ${ }^{4}$ Resident, Department of ENT, Head and Neck Surgery, AFMC, Pune.
}

\section{ABSTRACT}

\section{BACKGROUND}

A large number of Indian population stays in the desert area. The nature of nasal problems faced by them is mainly in the form of obstructive nasal symptoms, sicca syndrome, recurrent epistaxis and allergic features. The aim was to study the changes in nasal mucosa in individuals exposed to the extreme hot and dry weather conditions in the Desert Sector. It was felt that the personnel of the Paramilitary and Military Forces not exposed to these conditions were the ideal subjects to study such effects of the hot and dry weather conditions.

\section{MATERIAL AND METHODS}

A prospective observational study was done amongst 90 healthy volunteers exposed to the hot and dry desert conditions for the first time. The clinical profile of such patients was analysed along with their nasal endoscopy findings and nasal biopsy at the very onset and on completion of the study. The mucociliary transit time was also measured in them at the onset of study and after a period of two months. Thereafter the volunteers were divided into two groups and one group administered normal saline nasal drops and also advised saline nasal douching, whereas no such advice was given to the other group. After a period of three months, mucociliary transit time was measured and repeat biopsy was taken from each group and the results compared.

\section{RESULTS}

The Nasal Mucociliary Transit Time (NMTT) was significantly prolonged after two months stay in hot and dry weather conditions of desert $(p<0.05)$ along with a significant derangement in the mucosal lining in histopathological finding in the study population. Normal saline nasal drops were found to be successful to improve the nasal mucociliary transit time significantly $(\mathrm{p}<0.05)$.

\section{KEYWORDS}

Epistaxis, Atrophic Rhinitis, Mucociliary Transit Time, Sinusitis.

HOW TO CITE THIS ARTICLE: Singh SK, Mokamati S, Kanzhuly MK, et al. Nasal mucosa and mucociliary changes in extreme hot and dry weather in the deserts. J. Evolution Med. Dent. Sci. 2016;5(29):1483-1487, DOI: 10.14260/jemds/2016/349

\section{INTRODUCTION}

Deserts cover about one-fifth (20 percent) of the earth's land area. The arid Thar Desert is the world's seventh largest desert and is without doubt the most inhospitable ecoregion in the Indo-Pacific region. The climate is extreme: annual temperatures can range from near-freezing in the winter to more than $50^{\circ} \mathrm{C}$ during the summer.

Effects of hot and dry weather conditions on the nasal mucosa and its ciliary clearance is an area, which has remained grossly unexplored. Most of the reports and knowledge in this field is anecdotal and no proper study exists more so in the setting of hot and dry desert sector well known for its peculiar climatic conditions. It is amongst such peculiar environment that our troops are deployed. Troops from the military and paramilitary forces man most of the out-posts all the year round braving the heat, exhaustion and dryness. The soldier deployed here is not only guarding the border, but is also fighting the vagaries of nature.

Financial or Other, Competing Interest: None.

Submission 19-02-2016, Peer Review 17-03-2016,

Acceptance 22-03-2016, Published 11-04-2016.

Corresponding Author:

Dr. Sanajeet Kumar Singh,

Professor,

Department of ENT, Head and Neck Surgery,

Armed Forces Medical College,

Pune, India.

E-mail: sanajeetsingh@yahoo.in

DOI: $10.14260 /$ jemds/2016/349
A stuffy nose and bleeding from the nose are well known teething problems amongst people posted here. Apart from adversely affecting the health status of the soldier, these conditions also have a negative impact on his morale. Therefore, there is a requirement for addressing such problems in troops apart from other serious problems. The nature of nasal problem is mainly in the form of obstructive nasal symptoms and recurrent epistaxis. These problems lead to significant morbidity and loss of man-hours. The factors that contribute to these problems remain an enigma even today. Most of the reports and knowledge in this field is anecdotal and no proper study exists more so in the setting of hot and dry desert sector well known for its peculiar climatic conditions.

A clue into the causative factor can be inferred from a separate study by Salah B et al.[1] who demonstrated that breathing dry air at sea level reduces NMCT in healthy subjects. The low humidity and temperature further combine to deal with a severe blow to the nasal mucociliary motion, sometimes severely. [2] If dryness in addition to temperature be the cause of many of these symptoms, it was hypothesized that using of normal saline as nasal drops should show some amount of significant improvement in the nasal signs and symptoms. Therefore, a strong case exists for further analysing this hypothesis. However, before an assessment is done probing a possible intervention, it would be mandatory to establish in a scientific manner the very existence of nasal symptoms. 
Also by analysing the Nasal Mucociliary Transit Time (NMTT) delay, it may be possible to objectively assess the functional derangement of the nasal physiology. In a study Ciliary Beat Frequency (CBF) was also measured by fast Fourier transform analysis of computerized microscopic photometry.[3] CBF increased in an approximately linear fashion at $0.6 \mathrm{~Hz} /$ degree $\mathrm{C}$ between 20 degrees $\mathrm{C}$ and 35 degrees $\mathrm{C}$. Above 35 degrees $\mathrm{C}$, the increase was lower. Up to 44 degrees $\mathrm{C}$ all changes were reversible, while irreversible slowing and deterioration occurred above 45 degrees $\mathrm{C}$. In another study, a linear increase in CBF between 19 degrees and 32 degrees $\mathrm{C}$ was found. Between 32 degrees and 40 degrees a plateau was reached in which temperature did not significantly affect frequency, but above 40 degrees $C$ the frequency began to decline. The exposure to dust also alters the CBF.[4]

However, when assessing the problems faced by subtler diseases, classical mortality and morbidity data fail to assess the true hardships faced by the population. To scientifically analyse such a disease then becomes a challenge. The issues requiring attention in the present project were thus identified. These included studying the nature of nasal obstructive symptoms, their frequency, the type of symptoms and the nasal mucosal changes as seen clinically endoscopically and subsequently proved on biopsy.

An assessment of the derangement of nasal mucociliary transit times would enable us to actually measure the altered nasal physiology. The effect of using normal saline as nasal drops on the function of the nasal mucosa and possible improvement in the symptoms needed investigation and was incorporated by taking the biopsy at the conclusion of the followup.

\section{AIM AND OBJECTIVES \\ AIM}

To study the nasal mucosa and mucociliary changes in subjects exposed to hot and dry weather in the Desert Sector.

\section{OBJECTIVES}

1. To prospectively study the changes in nasal mucosa and the effect of extreme hot and dry weather as seen clinically and using nasal endoscopes.

2. To determine the derangement if any of Nasal Mucociliary Transit Time amongst troops after two and five months of induction into desert sector.

3. To study the effect of using Normal saline as nasal drops and saline nasal douching in averting the effect of heat and dryness and its role in improving the condition and function of the nasal mucosa.

4. To study the Histopathological changes in the nasal mucosa.

\section{MATERIALS AND METHODS}

The study was carried out at secondary care centre at Jodhpur. Jodhpur is not only an administrative and commercial centre, but also a pivotal hub for medical activities having a medical college. It is also a pivotal centre for military activities. To achieve our present objectives, we conducted two parallel activities. A longitudinal study was designed and implemented to study all the objectives.

\section{Study Population}

The study population included 90 healthy serving personnel freshly inducted into the desert sector. All subjects were taken from the units newly inducted in the sector and could be available for followup. The study was carried out during summer (Apr-Sep).

\section{Inclusion Criterion}

90 male serving personnel were randomly selected from the unit medical inspection room as they came for medical examination within a period of 01 month. A prior ENT disease/history suggestive of chronic rhinitis/allergic rhinitis formed an exclusion criterion. Any subject having significant Deviated Nasal Septum was also excluded from the study.

All subjects were counselled about the nature of the study and informed consent taken, especially for the Nasal Biopsy. All subjects were given the freedom to withdraw from the study at any time if they so wished. Institutional ethical clearance was taken prior to conduct of the study.

\section{Study Protocol}

On fulfilling the inclusion criterion and registration, the subjects underwent a detailed baseline evaluation including NMTT and Nasal Biopsy. The Nasal Biopsy was done in the OPD under local anaesthesia. At two months, the individual was again examined and evaluated. At this stage the subject was randomly allotted one of the two groups - Group A and Group B. Group A was asked to administer Normal Saline nasal drops regularly along with saline nasal douching twice daily and Group B was not given any treatment or intervention. The subjects were reviewed after three and five months. SPSS software was used for statistical analysis and paired ' $t$ ' test used or test of significance. The overall study protocol is as depicted in Table 1.

\section{Study Design}

The study was a prospective observational study. After three months, a non-blinded randomized trial comparing the efficacy of Normal Saline nasal drops in reversing these changes was done. The changes in the nasal mucosa were compared between the subjects given the saline nasal drop and those not given any treatment.

\section{NMTT}

The NMTT or the saccharin test was done in all subjects, wherein a particle of commercially available medical grade saccharin sweetener was used. It was placed approximately 1 $\mathrm{cm}$ behind the anterior end of the inferior turbinate on the nasal floor. The average time for sweet taste noticed was noted on each side separately at intervals of 30 minutes and average time was noted.

\section{Randomisation}

After two months of continuous stay, all the subjects who returned were further randomly allocated into two groups using computerised randomisation. Group ' $A$ ' was given Normal Saline nasal drops and advised to use them regularly. A dose of five drops three times a day was prescribed. The method of putting nasal drops was demonstrated to the individual. In Group ' $\mathrm{B}$ ', no normal saline drops were administered and subjects told to return after three months for the final examination. 


\section{Invasive Procedure}

Nasal mucosal biopsy was done at the onset and on the completion of the study, i.e. after 05 months. Nasal endoscopy was done at all the visits. No additional/invasive procedure was done for the purpose of the study.

\section{RESULTS}

The study subjects were in the age group of 24-45 years. They had no history of any ENT problem. After a detailed history and ENT evaluation, only those subjects who were apparently free from any ENT disorder were included in the study. The protocol and followup of these subjects is depicted in Table 1.

\section{Initial Evaluation}

Baseline evaluation was done for all subjects enrolled in the study as described in the materials and methods. NMTT was calculated for each individual. Nasal biopsy was done only in 60 subjects, as it was an invasive procedure and required their informed consent to do this.

\section{After 2 Months \\ History and Physical Examination}

82 subjects reported and were available for evaluation after 2 months. The symptoms suffered by the subjects were gauged by history taking. Out of the subjects who came back 33 (40\%) responded positively to suffering from some or the other nasal symptoms routinely over the two months' period. A further 28 $(34 \%)$ occasionally suffered from nasal symptoms in the last two months. However, 21 (26\%) of the subjects were apparently symptom-free. The frequency of nasal symptoms suffered by our subjects is depicted in Fig. 1. A breakup of the symptoms of the subjects is given as in Fig. 2. Nasal discharge and loss of smell were less commonly reported by the patients.

The physical findings on examination were graded as normal, mild, moderate or severe mucosal changes by OPD nasal endoscopy. Only 09 subjects (11\%) had a normal mucosa. Severe mucosal changes were found in 14 subjects (15\%) of the subjects. In most of the subjects, there were mildto-moderate changes. The results are as shown in the Fig. 3.

Around 23 of the subjects had a mucosal breach and a raw surface was visible. A dry and pale mucosa and mucoid nasal discharge were other commonly seen findings. The physical findings as seen by nasal endoscopy are as depicted in Figure 4.

\section{Nasal Mucociliary Transit Times (NMTT)}

To demonstrate the derangement of NMTT, mean transit times of subjects who reported at 2 months' followup interval were compared with their baseline values $(n=82)$. The mean NMTT of these subjects on induction was 7.9 minutes ( $\mathrm{SD}=2.32$ ). After a continuous stay of two months, the mean duration had increased to 15.8 minutes (SD=2.85) (See Figure 5).

The paired ' $\mathrm{t}$ ' test was applied to these measurements, which found the increase in NMTT to be statistically significant $(p<0.05)$. To study the effect of administering normal saline nasal drops to the NMTT, the subjects were randomly divided into groups as explained in the material and methods.

32 subjects of the A Group and 29 subjects of the B Group came for a further followup examination after 3 months. The mean NMTT of Group A, who were administered saline had improved to 12.15 minutes $(\mathrm{SD}=2.08)$, whereas the mean NMTT in the control Group B improved only to 14.2 minutes $(\mathrm{SD}=2.61)$.
The mean decrease in the transit time was 3.65 minutes in the study group, whereas in the control group it reduced by 1.6 minutes (Figure 6). Paired ' $t$ ' test was applied to see the reduction in transit times in Group A, which was statistically significant $(\mathrm{p}<0.005)$. There is a statistically significant improvement in the NMTT to the tune of 2.85 minutes to 6.1 minutes after regular administration of normal saline nasal drops.

\section{Histopathological Findings}

Nasal biopsy was done in 60 cases at the onset of the study. The initial biopsy showed a normal study in 56 cases, the significance of which could not be commented upon as a similar study is required for comparison at a normal environment. The repeat biopsy on the conclusion of the study could be done only in 08 cases of A and 06 cases of B study group. This was so because the biopsy was done $2^{\text {nd }}$ time in the same subjects and at this time there was extreme resistance to this invasive procedure. The repeat biopsy showed few metaplastic changes and features of chronic inflammation in 03 cases of A and 04 cases of B study group. Due to the curtailed sample size, the statistical significance cannot be commented upon.
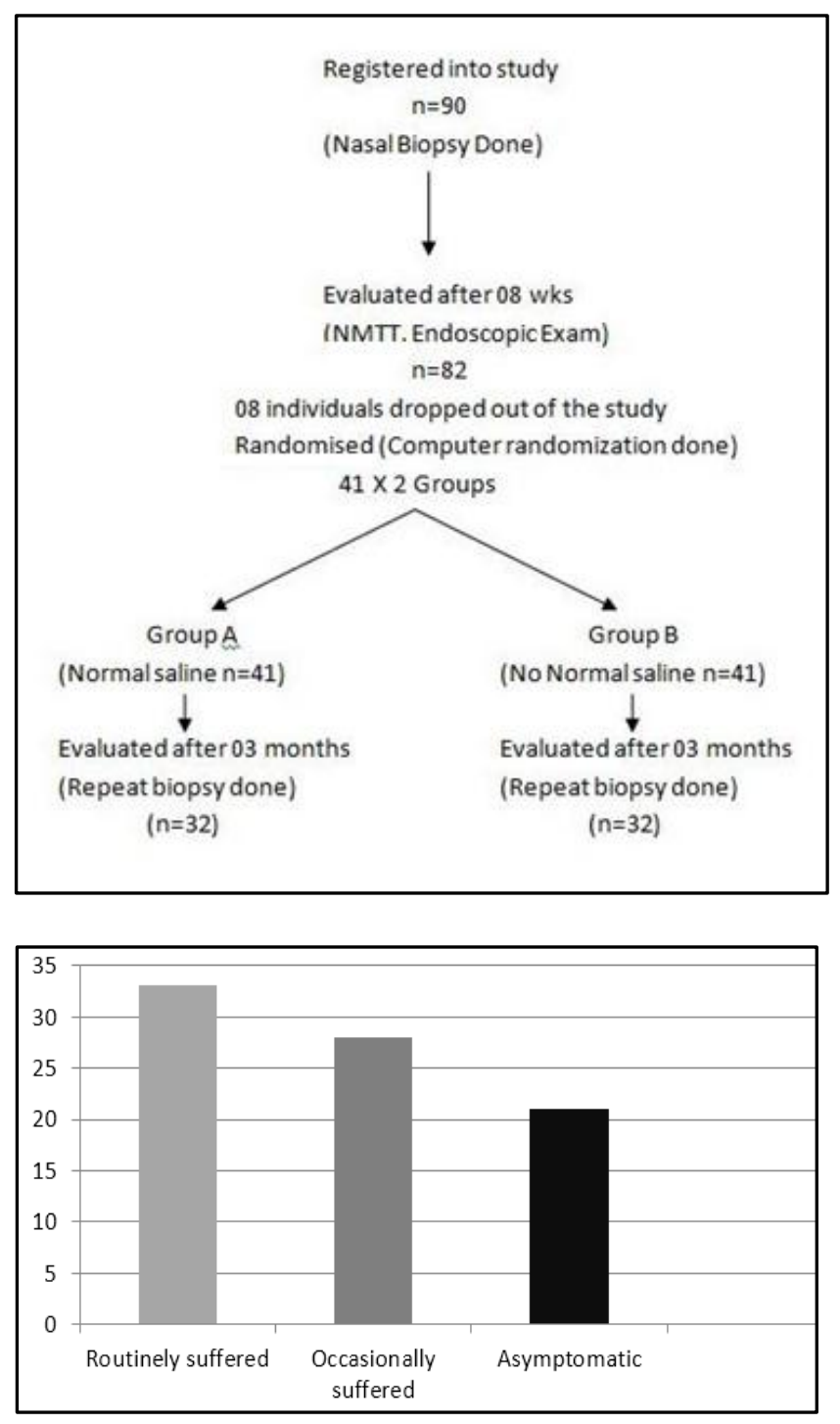

Fig. 1: Frequency of Nasal Symptoms in Subjects after 2 months at Desert Sector 


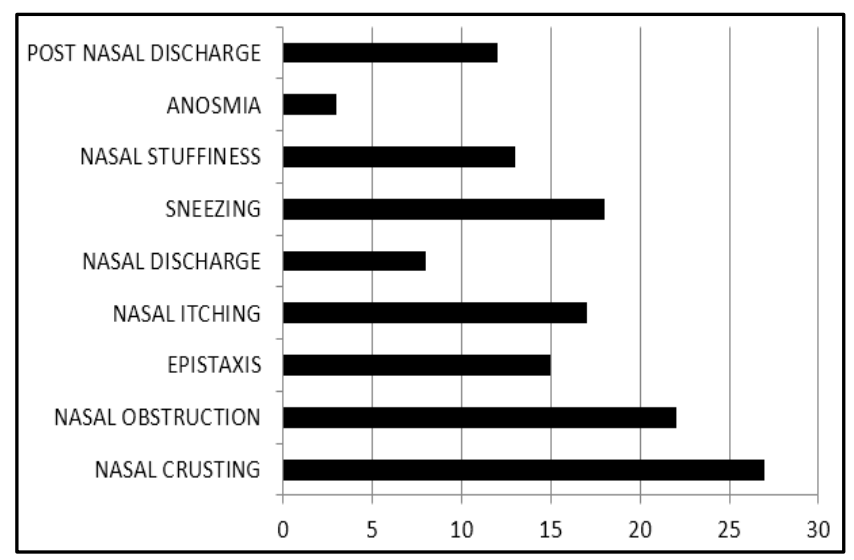

Fig. 2: Symptom profile in \% after 2 months of stay at Desert Sector

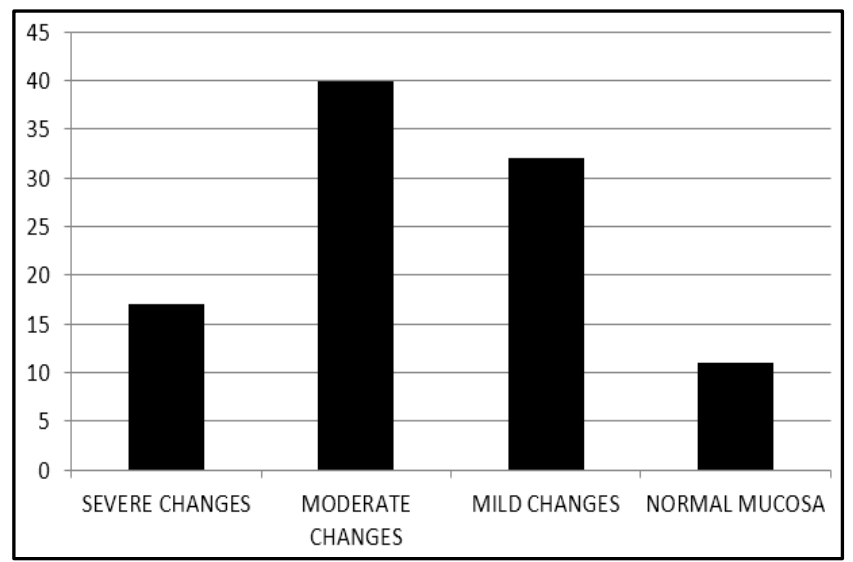

Fig. 3: Nasal Mucosal changes after 2 months stay at Desert Sector

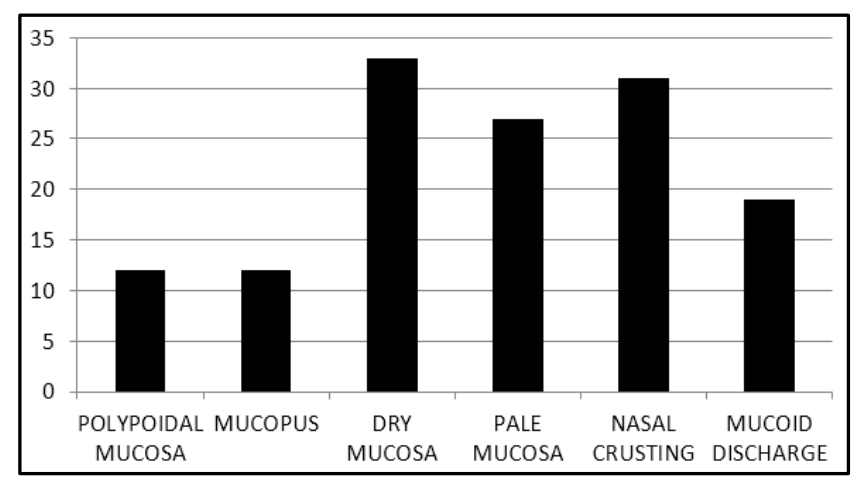

Fig. 4: Endoscopic findings after 2 months of stay at Desert Sector n=82 (Percentage) Polypoid mucosa-12\%,

Mucopus - 12\%, Dry mucosa - 33\%, Pale mucosa - 27\%, Nasal Crusting - 31\%, Mucoid discharge - 19\%

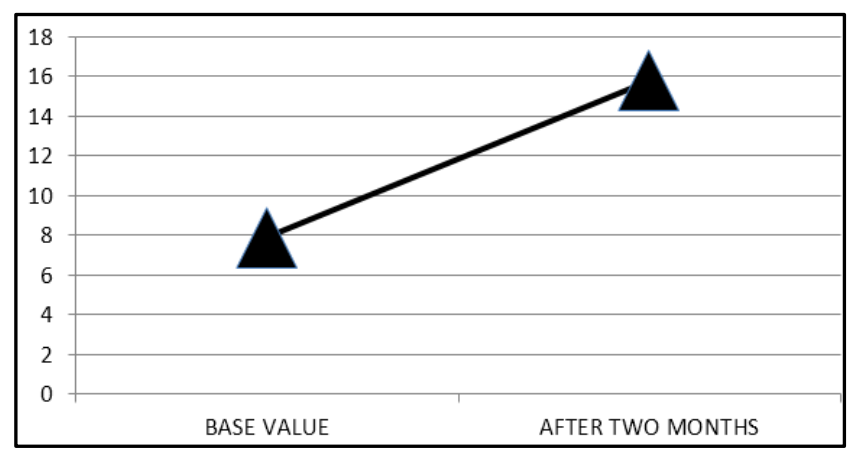

Fig. 5: Mean Nasal Mucociliary Transit
Times (Mean \pm SEM)

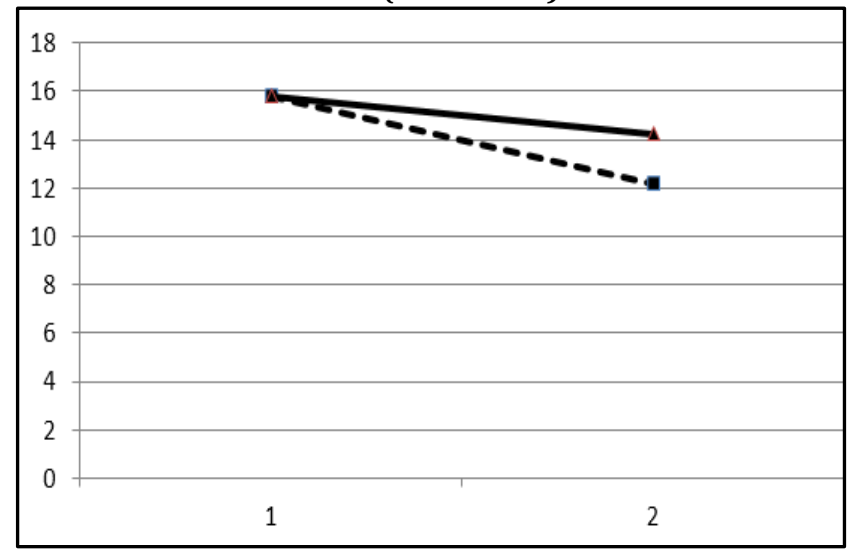

Fig. 6: Nasal Mucociliary Transit Times in Group A (Dotted line) and B (Continuous line) after 5 months in Desert Sector (Mean \pm SEM)

\section{DISCUSSION}

Nasal Symptoms

Over $74 \%$ of our subjects had suffered some or the other nasal symptoms at some point of time. The appearance of nasal symptoms in such a large proportion only highlights the widespread nature of such problems. More so these symptoms appeared in apparently healthy, previously asymptomatic individuals.

\section{Nasal Endoscopy Findings}

A large proportion of the subjects $(72 \%)$ had mild-tomoderate changes on nasal endoscopy with preponderance towards the anterior aspect. Hot and dry desert environment has lesser impact on the key osteomeatal complex area and ethmoid complex.

\section{Nasal Mucociliary Physiology}

The nasal mucosa forms an initial barrier to the onslaught of the hot and dry atmosphere of desert and understandably its physiology is altered as an effect. Approximately, 2 litres of secretions is produced daily from the goblet cells, seromucinous glands and lacrimal glands. The regulation of secretion depends on autonomic function, neuromediators and environmental factors.

The entire nasal cavity and paranasal cavity is covered with a homogeneous mucous blanket, which changes 2-3 times each hour and has an average speed of $6-10 \mathrm{~mm} / \mathrm{minute}$. It moves in anterior to posterior direction in the nose and towards the natural ostium in paranasal sinuses. It consists of a double layer, namely the outer 'gel' layer and inner 'sol' layer.

The beating of the cilia is the most important mechanism responsible for mucociliary clearance. The ciliary beat cycle takes place 10-20 times per second at normal body temperature and this can vary with the consistency of nasal secretions.

\section{Nasal Mucociliary Transit Time}

In a study comparing three of the common methods to judge the efficacy of the nasal mucociliary mechanism, Puchelle et al. validated the suitability of using saccharin as a simple screening test for nasal mucociliary clearance times. ${ }^{[5]}$ The mean NMTT had increased to 15.8 minutes from 7.9 minutes $(n=82)$. It can be stated that an exposure of hot and dry desert environment increases the nasal mucociliary transit time by approximately 7.8 minutes at two months. 
The most plausible reason should factor the peculiar environmental condition of hot and dry desert environment. The most obvious change in the environment is the high and dry temperature. The nasal cilia are sensitive to high temperatures and the ciliary beat frequency is known to reduce at higher temperatures.[6] The air in this area is dry and this may be another important factor. Another factor contributing to the derangement of NMTT is presence of nasal crusts and nasal obstruction due to it. Nasal obstruction in itself is known to alter the mucociliary transit times. Deitmer suggested a decoupling of the mucociliary transport leading to prolongation of NMTT following nasal obstruction. [7]

It is likely that prolongation of nasal mucociliary time in hot and dry desert environment is multifactorial in nature with important factors being the hot temperature, dry air and nasal obstruction. The decrease in the transit time in the study, Group A was statistically significant implying a noteworthy improvement in the function of the nasal mucosa. Given that the pathogenesis of altered nasal physiology is eventually a breakdown in the mucociliary transport mechanism, a restoration of the system should also reverse the symptoms.

Normal saline nasal drops are commonly available over the counter drugs and can be used, especially in paediatric population. Used along with specific treatment, it forms the cornerstone of managing chronic rhinosinusitis.[8] It probably serves to restore the sol phase of the dehydrated mucociliary blanket and dampens the crust formation. However, even though saline may be able to restore the water content of the mucus, it does not counter the factors of heat and dust which in themselves may cause delayed transit times.

\section{CONCLUSION}

The nasal mucosa in hot and dry desert environment is definitely altered, as it bears the brunt of the cold, dry atmospheric air which is peculiar in this region. There are some scientific conclusions, which can be drawn from this study. All the conclusions should be applied only to the target population.

1. Suffering from nasal symptoms is common amongst troops deployed in hot and dry desert environment.

2. There is an increased mucociliary transport time after exposure to hot and dry desert environment denoting a deranged physiology of the nasal mucosa.

3. The use of Normal Saline nasal drops is a simple and effective method of reversing some of the nasal physiological changes and the quality of life in these areas.
4. The nasal mucosa showed signs of inflammation and metaplasia after chronic exposure.

5. The peripheral medical officers are aware of this problem but have been using decongestant nasal drop, which can cause more harm and topical cream in terms of Ghee or Vaseline which is of limited use.

6. The observations were corroborated by the ENT Department of Jodhpur Medical College, where however no study was carried out but the day-to-day OPD followed almost the same pattern.

7. The epistaxis in the hot and dry weather also had an increased incidence in the paediatric age group and most of them responded very well to the Saline nasal drop.

8. There is a research institute, Desert Medicine Research Centre in Jodhpur, but the main interest of their research has been on Silicosis and Tuberculosis. No similar research was undertaken by them.

9. There has been a serious paucity of reference and research on this subject. Most of the research has been on the animals in desert, but not on humans.

\section{REFERENCES}

1. Salah B, Dinh Xuan AT, Fouilladieu JL, et al. Nasal mucociliary transport in healthy subjects is slower when breathing dry air. Eur Respir J 1988;1(9):852-5.

2. Riechelmann, Herbert MD, Rettinger, et al. Short term exposure to urban dust alters the mediator release of human nasal mucosa. Journal of Occupational and Environmental Medicine 2004;46(4):316-22.

3. Jorissen $M$, Bessems A. Influence of culture duration and ciliogenesis on the relationship between ciliary beat frequency and temperature in nasal epithelial cells. European archives of oto-rhino-laryngology. 1995;252(8):451-454.

4. D'Amato G, Liccardi G, D'amato $M$, et al. Outdoor air pollution, climatic changes and allergic bronchial asthma. European Respiratory Journal 2002;20(3):763-76.

5. Puchelle E, Aug F, Pham QT, et al. Comparison of three methods for measuring nasal mucociliary clearance in man. Acta Otolaryngol 1981;91(3-4):297-303.

6. Green A, Smallman LA, Logan AC, et al. The effect of temperature on nasal ciliary beat frequency. Clin Otolaryngol 1995;20(2):178-80.

7. Deitmer T, Erwig H. The influence of nasal obstruction on mucociliary transport. Rhinology 1986;24(3):159-62.

8. Aukema AA, Fokkens WJ. Chronic rhinosinusitis: management for optimal outcomes. Treat Respir Med 2004;3(2):97-105. 